\title{
Simulation of Electromagnetic Coupling on Pipelines close to Overhead Transmission Lines: A Parametric Study
}

\author{
A. H. Al-Badi and H. M. Al-Rizzo
}

\begin{abstract}
Electromagnetic interference effects caused by electric power lines on neighboring metallic utilities such as water, gas or oil pipelines became a major concern due to significant increase in the load and short circuit current levels needed to satisfy the load requirements. Another reason for increased interference levels originates from the environmental concerns, which impose on various utilities the obligation to share common corridors. This paper presents three different scenarios of a pipeline in which all types of electromagnetic interferences (coupling) will be investigated and their effects on the pipeline will be predicted. The level of the calculated voltage, owing to each type of coupling, depends on different factors (voltage level, length of parallelism, separation distance, soil resistivity, load current magnitude and pipeline coating). The effects of these factors are discussed; some factors such as the fault current level, separation distance and soil resistivities are found to exhibit a large influence on the pipeline voltage. To the best of the authors' knowledge, the comprehensive analyses presented in this paper considering all types of interferences have not yet been published elsewhere.
\end{abstract}

Index terms: Pipeline, Power line, Inductive coupling, Conductive coupling, Capacitive coupling

\section{INTRODUCTION}

Oil/gas/water pipelines and overhead power lines share the same right-of-way in some areas. As a consequence, these pipelines can incur high induced voltages and currents due to the AC interference. Magnetic and electric fields surrounding the power system in air and soil energize the pipeline. The induced voltage on pipelines can be dangerous for an operator as well as pipe corrosion can result from AC discharge [1-6].

A potential shock hazard exists when someone touches an exposed part of the pipeline while standing on soil, which is at

Manuscript received July 18, 2005; revised September 25, 2005, and December 19, 2005. The research was supported in part by Sultan Qaboos University (SQU) and Petroleum Development Oman (PDO), Sultanate of Oman, in 2005.

A. H. Al-Badi is with Electrical \& Computer Engineering Department College of Engineering, Sultan Qaboos University, Sultanate of Oman (email: albadi@squ.edu.om)

H.M. Al-Rizzo is with System Engineering Department Donaghey College ofInformation Science and Systems Engineering, University of Arkansas at Little Rock, Little Rock, AR 72204-1099, USA (e-mail: hmalrizzo@ualr.edu) a different potential. Excessive coating stress voltages (the difference between the pipe steel potential and local soil potential) can lead to degradation of the coating, resulting in an accelerated corrosion. To rectify these problems, the pipelines must be grounded with a system that passes AC and blocks DC. The likelihood of interference increases with increasing overhead line current, with increasing soil resistivity, with decreasing the separation distance, with increasing quality of the coating on the pipeline, and with the length of pipeline parallel to and close to the transmission lines. The electromagnetic interference between a power system network and neighboring pipeline has been traditionally divided into three categories: capacitive (electrostatic), conductive (resistive) and inductive (magnetic) coupling [3]. The first is the capacitive interference, which is generated by the electric field and occurs when the pipe is placed on a foundation that is well insulated from ground. The pipe picks up a voltage relative to soil that is proportional to the transmission line voltage. The second is the conductive interference, which occurs during lightning strikes or a phase to ground fault. When this occurs, a large voltage cone is created around the grounding system: as a result a voltage can get onto the pipeline through the pipe coating defects. The difference in potential between the pipeline and the surrounding earth due to current discharge into earth represents the conductive interference. The magnitude of the conductive interference is mainly a function of ground potential rise of transmission structure, soil resistivity, separation distance and size of grounding system. The third is the inductive interference, which is generated by the magnetic field and is present during both steady-state conditions and fault conditions when the pipe is placed close to three-phase overhead transmission lines.

At exposed pipeline appurtenances such as valve sites and metering stations, the maximum acceptable touch voltage, during normal operating conditions, according to NACE standard RP-01-77-95 [7] and to ANSI/IEEE Standard 80 safety criteria [8] is 15 volts for structures which may be contacted by unexpected workers and general public. Pipeline potentials with respect to local earth ranging from 15 to 65 volts are considered acceptable in different countries. During fault conditions, pipeline potentials with respect to local earth (i.e., touch voltages) are not to exceed the limit determined in accordance with ANSI/IEEE Standard 80-2000. In this case, with fault duration of $0.3 \mathrm{~s}$, soil resistivity of $100 \Omega . \mathrm{m}$, the 
permissible "safe touch voltage", according to ANSI/IEEE standard is $244.8 \mathrm{~V}$. The touch voltage limit could be increased by applying a layer of crashed rock. Coating stress voltages must be maintained sufficiently low to prevent arcing through pipeline coating. This typically occurs for coating stress voltages on order of $3-5 \mathrm{kV}$ or higher for modern coatings such as fusion bonded epoxy [9].

In this paper three different scenarios of a pipeline are presented in which all types of electromagnetic interferences (coupling) are investigated and their effects on the pipeline are analyzed. The induced voltage along the pipeline sections is computed under different system parameters. The basic input data to the model consists of power line and pipeline geometrical configuration, line conductor and pipeline physical characteristics including insulation and coating characteristics, environmental parameters such as soil characteristics, power source voltage, and equivalent source impedances.

\section{SYSTEM MODEL PARAMETERS}

The system under consideration consists of transmission lines and a neighboring pipeline. The total length of the transmission lines is $30 \mathrm{~km}$; the pipeline is placed at the central site with total length of $10 \mathrm{~km}$ and it is buried at a depth of $0.5 \mathrm{~m}$. The investigation reported in this paper is based on a state-of-the-art commercial computer aided-design tool, the theory of which is described in [11]-[14]. In particular, the analysis of electromagnetic interference between the $132 \mathrm{kV}$ overhead power lines and the neighboring gas/oil/water pipelines has been carried using the Right-ofWay and MALZ programs. These programs are integrated parts of the well-known CDEGS software [10]. The Right-ofWay was used to calculate the inductive and capacitive components while MALZ was used to compute the conductive part. We have generated several series simulations, based on the circuit diagram for the system presented in Fig. 1, by varying one parameter at a time through a range of values.

The interference levels can generally be calculated by using either a circuit approach or a field approach. The circuit approach usually offers more flexibility for long right-of-way shared by overhead transmission lines and pipelines. Computation of the interference effects in such right-of-way is a complex procedure because factors such as power line and pipeline electrical characteristics, electrical system parameters, soil characteristics and conductors layout must be taken into account. Moreover, the distances between all conductors, soil structures and conductor characteristics normally vary along the right-of-way. Thus in order to build a circuit model, a large number of line parameter calculations needs to be performed, which is a time consuming process. Furthermore, analysis of electromagnetic interference levels for fault conditions requires simulation of a fault on each tower along the right-ofway, which again is a time consuming process. The Right-ofWay program is especially designed to simplify and to automate the modeling of complex configurations involving transmission lines and other utilities such as gas/oil/water pipelines, communication lines and railways [11]. All the relevant parameters used for modeling the system are taken into account in computing the line parameters, building the circuit model and automatically determining the maximum voltage levels under steady-state and fault conditions at all required location a long the right-of-way. However, the predictions from the circuit approach are always conservative [11]. In the circuit model method, one of the assumptions made for the computation of the line parameters is that the conductors are parallel to one another, which can lead to inaccuracy in the computed results [12].

The filed approach, on the other hand, is based on numerical solution of Maxwell's equations [13-14].

Numerical evaluation (Gaussian integration method) of Sommerfeld integrals is used instead of an analytical approximation in order to get highly accurate results. This approach models the complete conductor network under consideration, in three-dimensional space and accommodates angled conductors without making any approximations. The inductive, conductive and capacitive coupling effects between all the elements in the network are simultaneously computed in one single step. The detailed discussion of the computation methods employed by the software utilized in our study is presented in references [13] and [14]. Our study was performed using the CDEGS software package [10], which accommodates both the field and circuit-based model.

For the purpose of computing touch voltages in the vicinity of the pipeline, a long profile was specified which lying on the soil surface, right above the pipeline, starting at one end of the pipeline and ending at the other end with total length of 10000 m (see Fig. 1).

The following is a list of parameter settings of the computer models used in this study:

\section{Pipeline}

Coating Resistivity: 15665 S.m

Coating thickness: $0.005 \mathrm{~m}$

Outer Diameter: $0.4064 \mathrm{~m}$

Inner Diameter: $0.39923 \mathrm{~m}$

Relative Resistivity: 17 (with respect to annealed copper). Relative permeability: 250 (with respect to free space).

Grounding: None

\section{Overhead Transmission line}

\section{AAAC (single-ELM) $132 \mathrm{kV}$}

Geometric mean radius (G.M.R): $0.7122 \mathrm{~cm}$

Conductor outer radius: $0.94 \mathrm{~cm}$

Outer strand radius: $0.188 \mathrm{~cm}$

Number of strands: 19

Fault current (phase-to-ground fault), $\mathrm{I}_{\mathrm{f}}: 5 \mathrm{kA} \& 10 \mathrm{kA}$.

\section{System}

Length of parallelism: $10 \mathrm{~km}$

Soil Resistivity, $\rho: 100 \Omega . m$

Horizontal separation distance between pipeline and overhead transmission lines, $r=100 \mathrm{~m}$ 


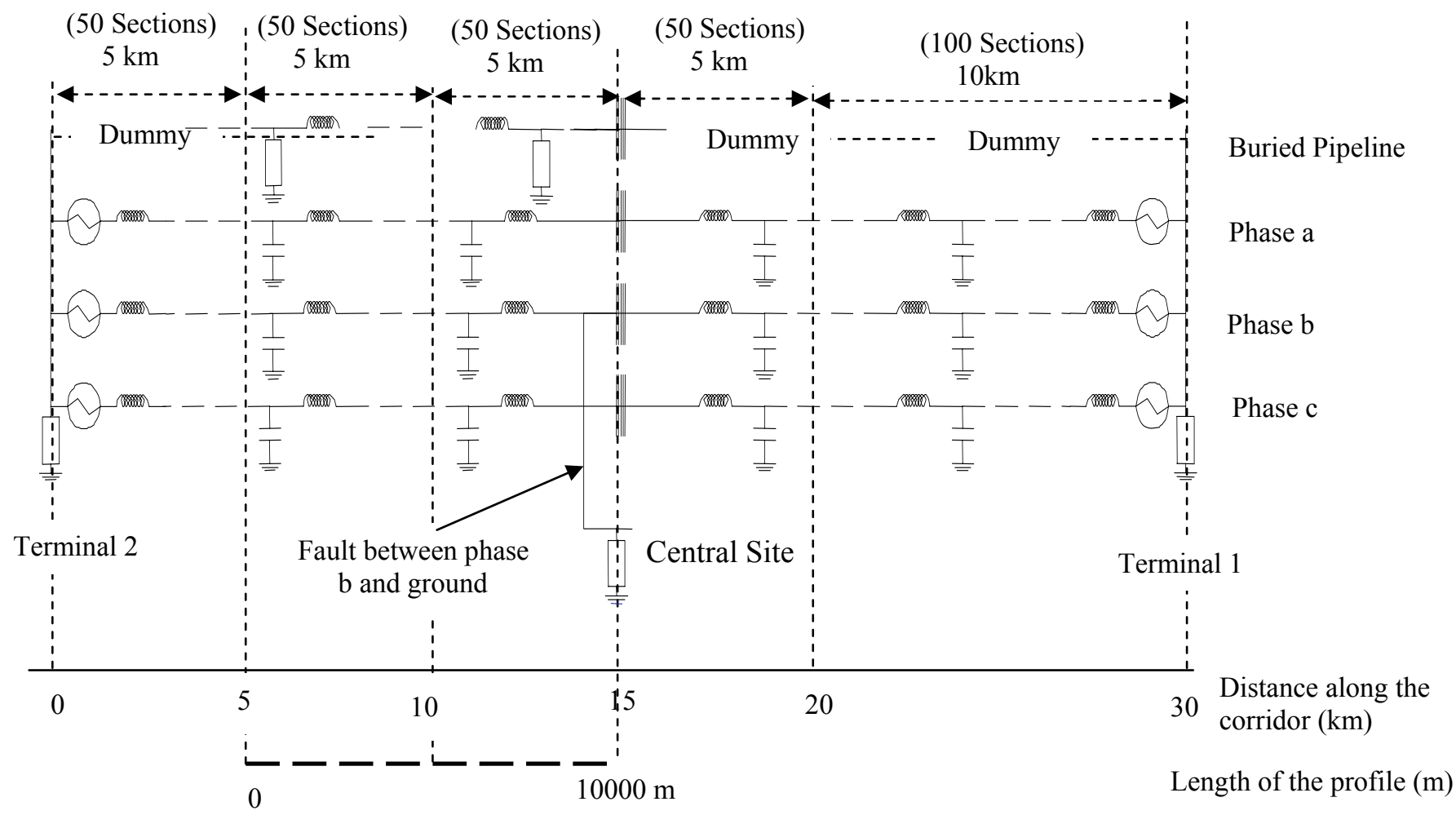

Fig.1: Circuit model for the case under study

\section{A. Definitions}

\section{Ground potential rise (GPR):}

The maximum electric potential that a grounding grid may reach relative to a distant grounding point assumed to be at the potential of remote earth. This voltage is equal to the maximum grid current times the grid resistance.

\section{Touch voltage:}

The touch voltage to which a person would be subjected when touching an exposed part of the pipeline is defined as the difference in potential between the pipeline metal and the earth surface where a person is standing.

\section{Coating stress voltage:}

The difference between the pipeline steel potential and the local soil potential.

\section{Steady state conditions}

Normal operating conditions of the electric power transmission system, which may vary from low to high load.

\section{Shunt Potential magnitude:}

The potential of the pipeline with respect to remote earth.

For the figures presented in this paper, the following should be noted:

- The touch voltage and shunt potential magnitudes are defined only when the pipeline is present along the corridor.

- For the conductive interference, the curve reaches a peak at tower location, where the current flows into ground.
- For the inductive interference, the shunt potential magnitude is symmetrical due to symmetry of the system. The minimum induced potential occurs at the center of the pipeline and the maximum induced voltage occurred at the begining and end of the pipeline because of the longitudinal current discontinuity.

\section{BURIED PIPELINE}

In this scenario a $10 \mathrm{~km}$ pipeline with burial depth of $0.5 \mathrm{~m}$ is considered to run in parallel with $30 \mathrm{~km}$ overhead transmission lines. The three types of interference are investigated at different factors. It should be noted that since the pipeline is buried, the effect of the capacitive coupling can be neglected.

\section{A. Effect of Fault Current Magnitude}

\section{A.1 Inductive Interference}

Based on phase fault currents $5 \mathrm{kA}$ and $10 \mathrm{kA}, 100 \mathrm{~m}$ separation (r), soil resistivity of $100 \Omega . \mathrm{m}$ and for $132 \mathrm{kV}$ power lines, the induced pipeline potential during phase to a ground fault condition is shown in Fig. 2. It can be noted that the pipeline-induced potential is very large and exceeding the standard limits. Therefore, increasing the fault magnitude will increase the induced voltage. It should be noted that the software generates a voltage profile along the whole length of the pipeline, in which the pipeline is divided into a number of sections and the length of each section is set to $100 \mathrm{~m}$. 


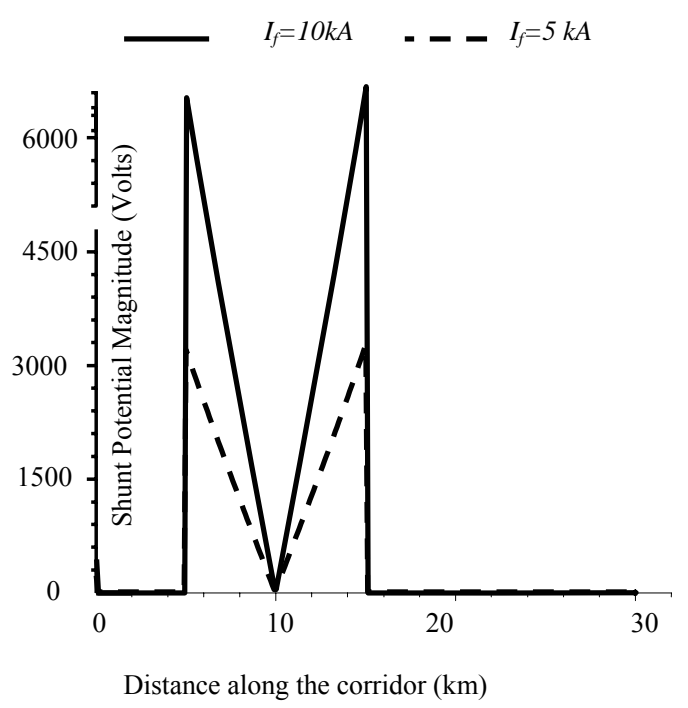

Fig. 2: Pipeline Potential for $\mathrm{r}=100 \mathrm{~m}, \rho=100 \Omega \mathrm{m}$ and for different values of $I_{f}$

Results obtained from the software [10] have been verified against the analytical approach reported in [15] and a good agreement has been obtained. The derivation is presented in Appendix A.

The maximum induced potential is given by the following formula:

$$
E_{f}=I_{\text {fault }} \cdot \frac{5}{8} \cdot \frac{f}{60}\left[0.0954+j 0.2794 \cdot \log \frac{D_{e X}}{D_{a x}}\right]
$$

where:

$E_{f}$ : The voltage induced in pipeline during the fault, $\mathrm{V} / \mathrm{km}$

$I_{\text {fault }}$ : The fault current, A

$D_{e x}$ : The depth of earth return path, $\mathrm{m}$

$D_{a x}$ : The separation between phase conductor and the pipeline, $\mathrm{m}$

The depth of earth return path is given as

$$
D_{\text {ex }}=660 \cdot \sqrt{\frac{\rho}{f}}
$$

The maximum induced potential is evaluated from:

$$
V_{\text {Max }}=\frac{E_{f}}{\gamma}
$$

where:

$\gamma$ is the propagation constant of pipe in $\mathrm{km}^{-1}$.

$\gamma=\sqrt{Z Y}$, where $Z$ is the pipe self impedance and $Y$ is the pipe shunt admittance per unit length.

A comparison between the analytical approach and our CDEGS-based model reveals that they are in a good agreement. The analytical approach, however, yields slightly higher results as shown in Fig. 3. It should be noted that the analytical approach reported in [15] neglects the current in the other two phases during the single line to ground fault.

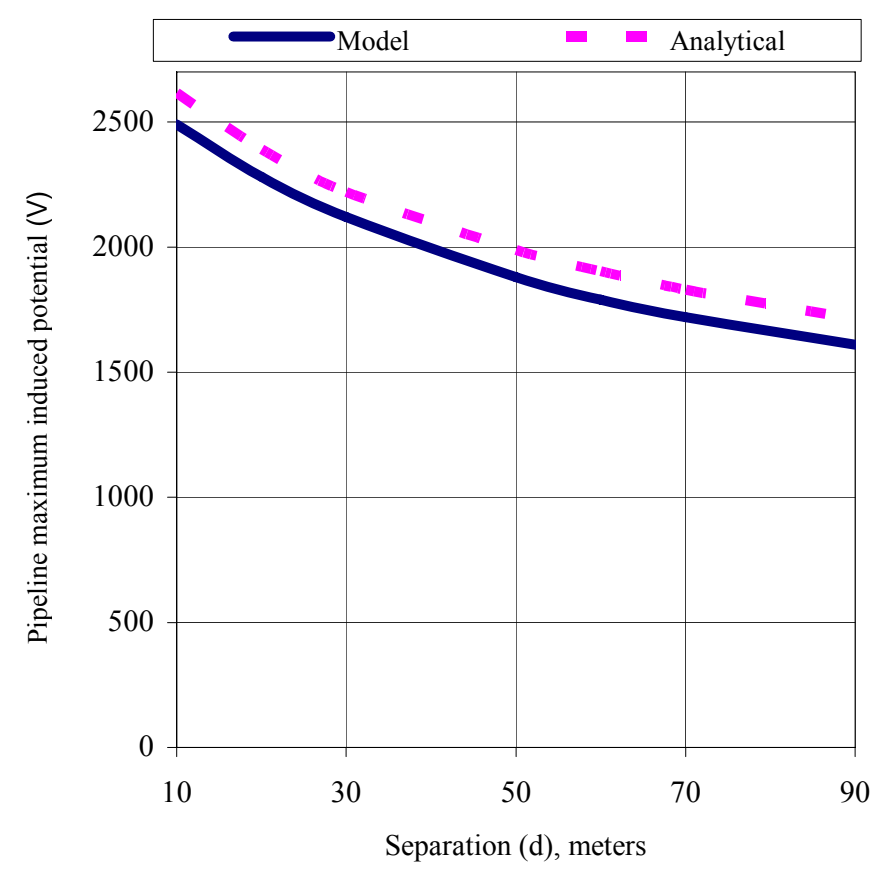

Fig. 3: Pipeline maximum induced potential, due to inductive interference under fault condition: $\mathrm{I}_{\mathrm{f}}=2000 \mathrm{~A}, 132 \mathrm{kV}, \rho=1000 \Omega . \mathrm{m}$ using analytical and CDEGS-based modeling.

\section{A. 2 Conductive Interference}

The pipeline voltage due to conductive interference is presented in Fig. 4, in which the touch voltage is calculated along a chosen profile as a function of the distance along the axial length of the pipeline. The contribution of the conductive component is smaller compared to that of the inductive one.

\section{B. Effect of Soil Resistivity}

Low soil resistivity means lower system ground impedance and lower potential differences between the grounding structure and the pipeline. We have analyzed the interference at different soil resistivities (the soil resistivity varied from 100 to $1000 \mathrm{ohm}-\mathrm{m})$. It is clear from Fig. 5 that the soil resistivity has an influence on the induced voltage during the fault current, in which the induced voltage is increased by 1.5 times. Moreover, the pipeline voltage increased by more than 9 times for the conductive effect, as illustrated in Fig.6.

\section{Effect of Separation distance}

Figs. 7 and 8 show how inductive and conductive interferences are affected by the changes in separation distance. 


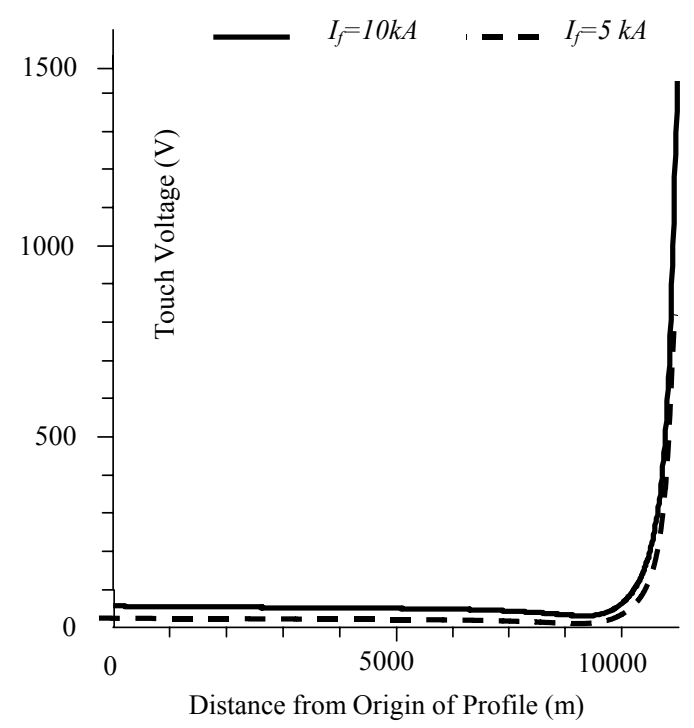

Fig. 4: Touch Voltage for $r=100 \mathrm{~m}, \rho=100 \Omega . \mathrm{m}$ and for different values of $I_{f}$

Naturally, the greater the separation distance between a pipeline and a nearby power transmission line, the lower will be the voltage on the pipeline. It is well known that the magnetic field produced by power lines during fault condition is proportional to the fault current flowing in the faulted phase conductors and inversely proportional with the separation distance between the power line and pipeline. Increasing the separation distance from $100 \mathrm{~m}$ to $600 \mathrm{~m}$ reduces the pipeline voltage, due to inductive effect, by $67 \%$, as presented in Fig. 7 , where as in the conductive component the voltage is reduced by $87 \%$ as shown in Fig. 8 .

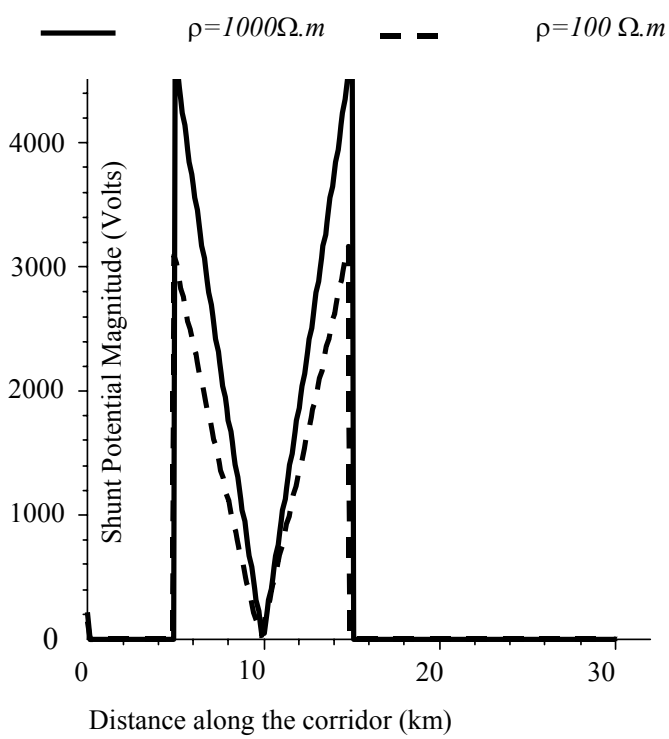

Fig. 5: Pipeline Potential for $\mathrm{r}=100 \mathrm{~m}, \mathrm{I}_{\mathrm{f}}=5 \mathrm{kA}$ and for different values of soil resistivities. (Inductive)

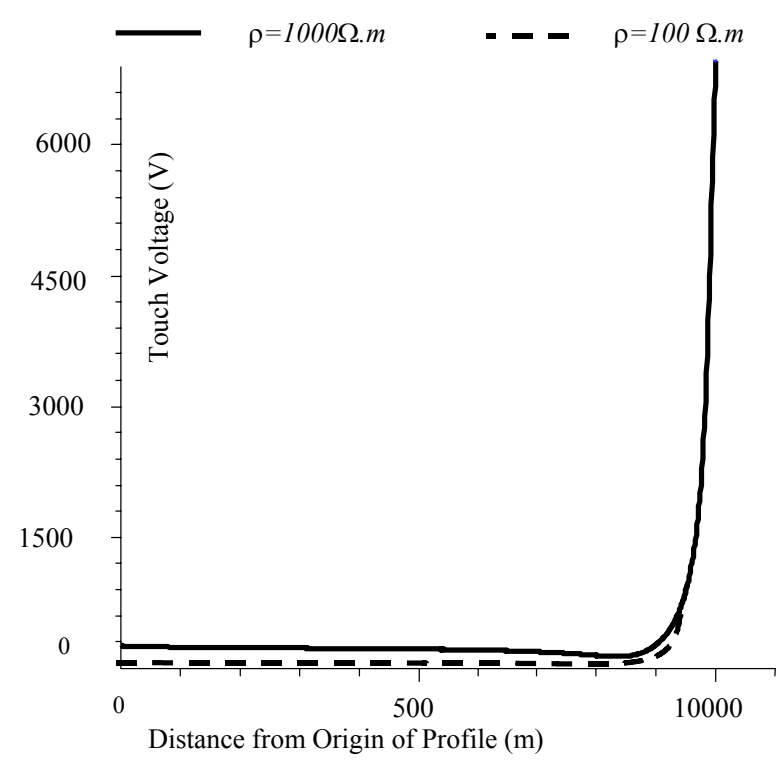

Fig. 6: Touch Voltage for $\mathrm{r}=100 \mathrm{~m}, \mathrm{I}_{\mathrm{f}}=5 \mathrm{kA}$ and for different values of soil resistivities. (Conductive)

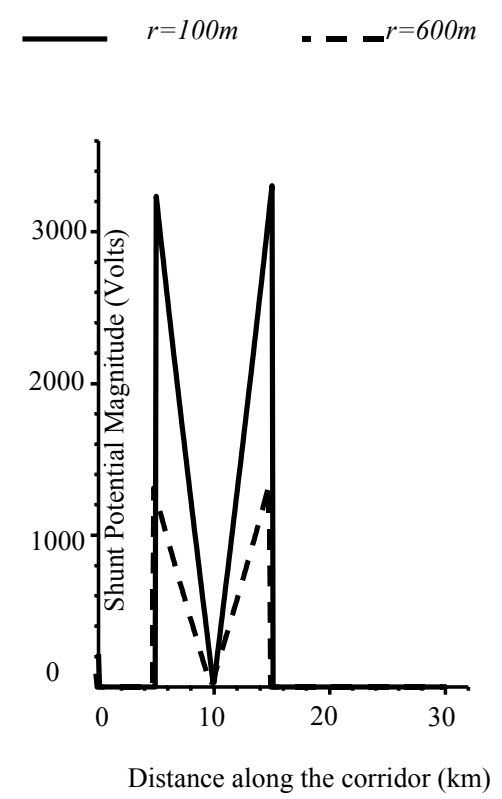

Fig. 7: Pipeline Potential for $\mathrm{I}_{\mathrm{f}}=5 \mathrm{kA}, \rho=100 \Omega$.m, and for different separation distances.

\section{Effect of length of parallelism}

The length of parallelism will affect mainly the inductive interference, as shown in Fig. 9. Reducing parallelism between 
overhead transmission lines and pipelines reduces the induced voltage, because the pipeline voltage depends on the length of the parallelism as shown in Equation (1). The value of the touch voltage (conductive interference) will not be affected by the parallelism as shown in Fig. 10.

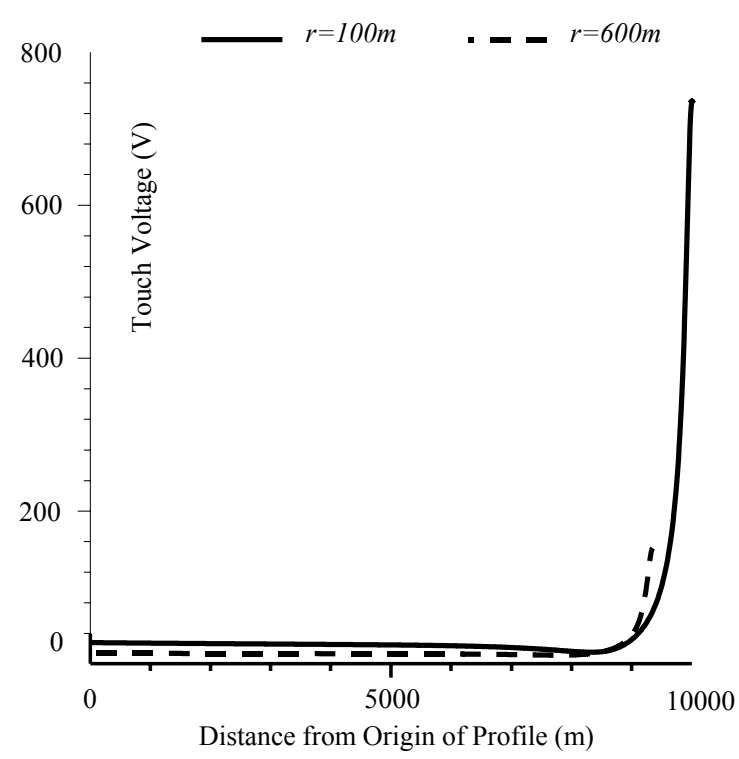

Fig. 8: Touch Voltage for $\mathrm{I}_{\mathrm{f}}=5 \mathrm{kA}, \rho=100 \Omega . \mathrm{m}$, and for different separation distances.

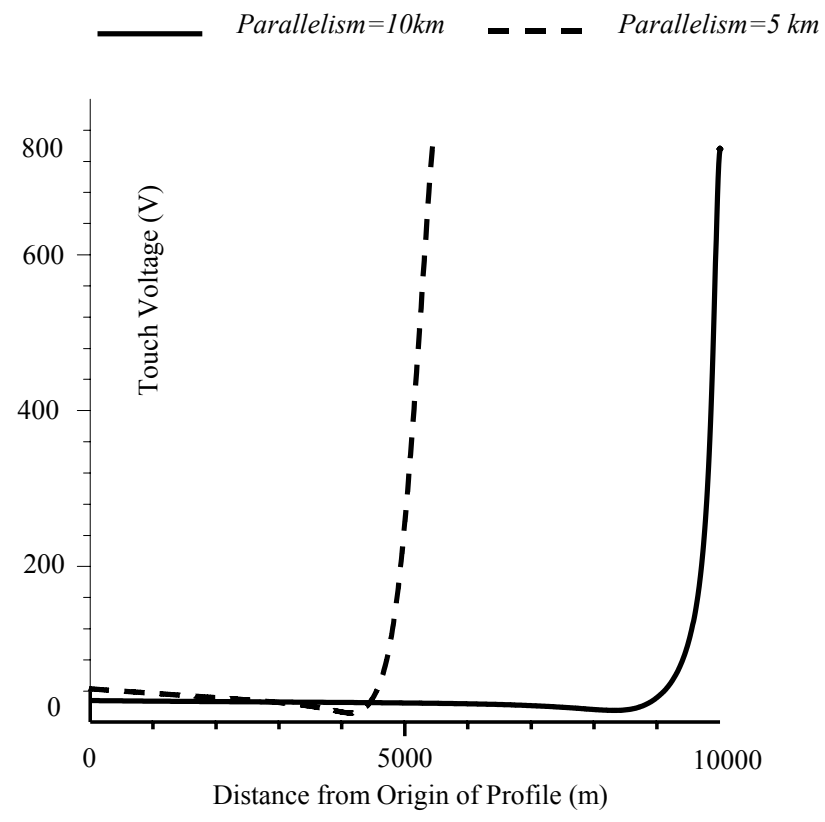

Fig.10: Touch Voltage for $\mathrm{r}=100 \mathrm{~m}, \mathrm{I}_{\mathrm{f}}=5 \mathrm{kA}, \rho=100 \Omega . \mathrm{m}$ and for different parallelism.

\section{E. Effect of Pipeline Coating Resistivity}

Parallelism $=10 \mathrm{~km} \quad \cdot \mathbf{-}-$ - Parallelism $=5 \mathrm{~km}$

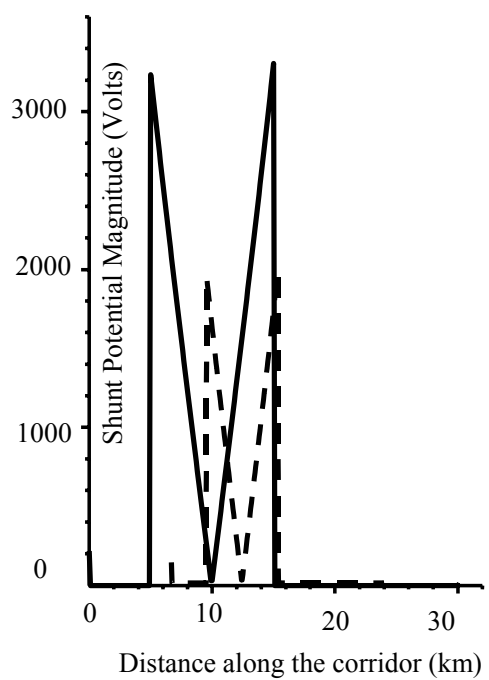

Fig.9: Pipeline Potential for $\mathrm{r}=100 \mathrm{~m}, \mathrm{I}_{\mathrm{f}}=5 \mathrm{kA}$, $\rho=100 \Omega . m$ and for different parallelism.
The inductive interference has been analyzed for different pipeline coating resistivities. It is clear that the coating resistivity has an influence on the induced voltage during the fault current. The better the coating, the higher the induced voltage as current does not easily leak from a well coated pipeline. The results for the inductive interferences at different coating resistivity are given in Table I. It is clear that the pipeline coating has a negligible effect on the conductive interference as depicted in Table I.

\section{TABLE I}

PIPELINE VOLTAGE FOR $132 \mathrm{KV}$ LINE, PIPE DIAMETER $16 ", \rho=100 \Omega$.m $\mathrm{I}_{\mathrm{f}}=5000 \mathrm{~A}, \mathrm{r}=100 \mathrm{~m}$, WITH DIFFERENT COATING RESISTANCES AND COATIN THICKNESS $=0.0036 \mathrm{~m}$

\begin{tabular}{|c|c|c|}
\hline $\begin{array}{c}\text { Coating } \\
\text { Resistance per } \\
\text { unit Area }\left(\Omega . \mathrm{m}^{2}\right)\end{array}$ & $\begin{array}{c}\text { Voltage (V) } \\
\text { (Inductive) }\end{array}$ & $\begin{array}{c}\text { Voltage (V) } \\
\text { (Conductive) }\end{array}$ \\
\hline 1000000 & 3700 & 760 \\
\hline 20000 & 3400 & 740 \\
\hline 10000 & 2800 & 730 \\
\hline
\end{tabular}




\section{PIPELINE ABOVE GROUND}

In this scenario a $10 \mathrm{~km}$ pipeline is considered which is run in parallel to a $30 \mathrm{~km}$ overhead transmission lines. The pipeline is maintained at a level of $0.5 \mathrm{~m}$ from the earth surface. The inductive coupling is found to be the same as the buried case. Moreover, there is no major change in case of conductive coupling as shown in Fig.11. The following will be the factors that affect the level of capacitive coupling.

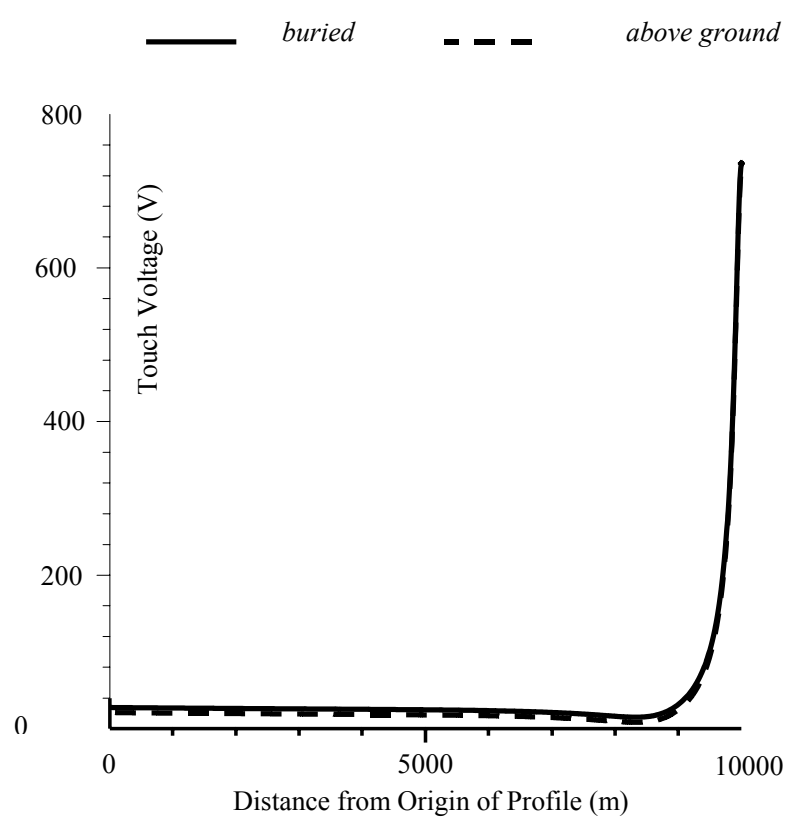

Fig. 11: Touch Voltage for $\mathrm{I}_{\mathrm{f}}=5 \mathrm{kA}, \rho=100 \Omega . \mathrm{m}, \mathrm{r}=100 \mathrm{~m}$ and pipeline buried and above ground.

\section{A. Applied voltage}

Based on applied r.m.s. voltages of $33 \mathrm{kV}$ and $132 \mathrm{kV}$, separation distance of $10 \mathrm{~m}$ and soil resistivity of $100 \Omega \mathrm{m}$, the pipeline potential is shown in Fig. 12. From this figure, it can be noted that the pipeline potential is large. Therefore, increasing the applied voltage magnitude will increase the induced voltage owing to the increase in the strength of the electric field.

\section{B. Soil Resistivity}

The interference effect has been analyzed at different soil resistivities (the soil resistivity varied from 100 to $1000 \Omega . \mathrm{m}$ ). It is clear that the soil resistivity has no influence on the induced voltage owing to the capacitive coupling as depicted in Fig. 13 because the pipeline is situated above the ground.

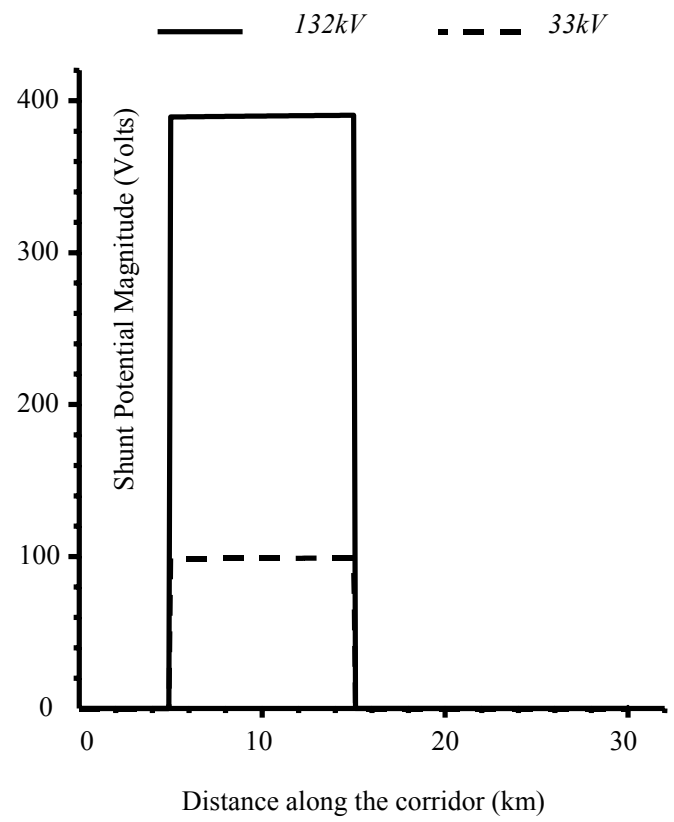

Fig. 12: Pipeline Potential for $r=10 \mathrm{~m}, \rho=100 \Omega . \mathrm{m}$ and for different applied voltage.

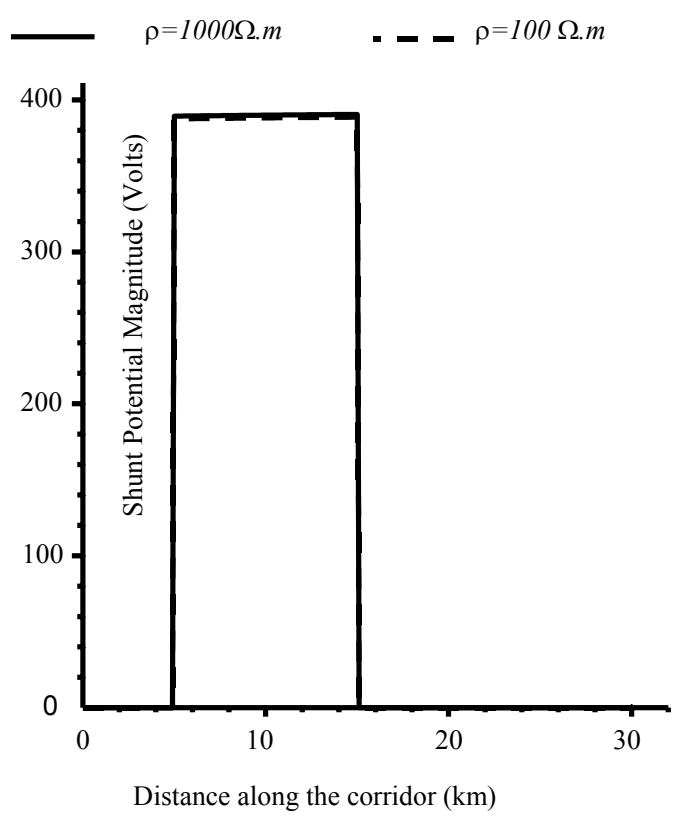

Fig. 13: Pipeline Potential for $\mathrm{r}=10 \mathrm{~m}, \mathrm{~V}=132 \mathrm{kV}$ and for different soil resistivities.

\section{Separation Distance}

The effect of changing the separation distance can be inferred from Fig. 14. Naturally, the greater the lateral distance between a pipeline and a nearby power transmission line, the smaller will be the strength of the electric field; and as a result the voltage induced on pipeline is lower. 




Fig. 14: Pipeline Potential for $\mathrm{V}=132 \mathrm{kV}, \rho=100 \Omega . \mathrm{m}$ and for different separation distances.

\section{Length of Parallelism}

The length of parallelism does not affect the capacitive coupling as shown in Fig. 15. The length of parallelism affects only the inductive coupling.

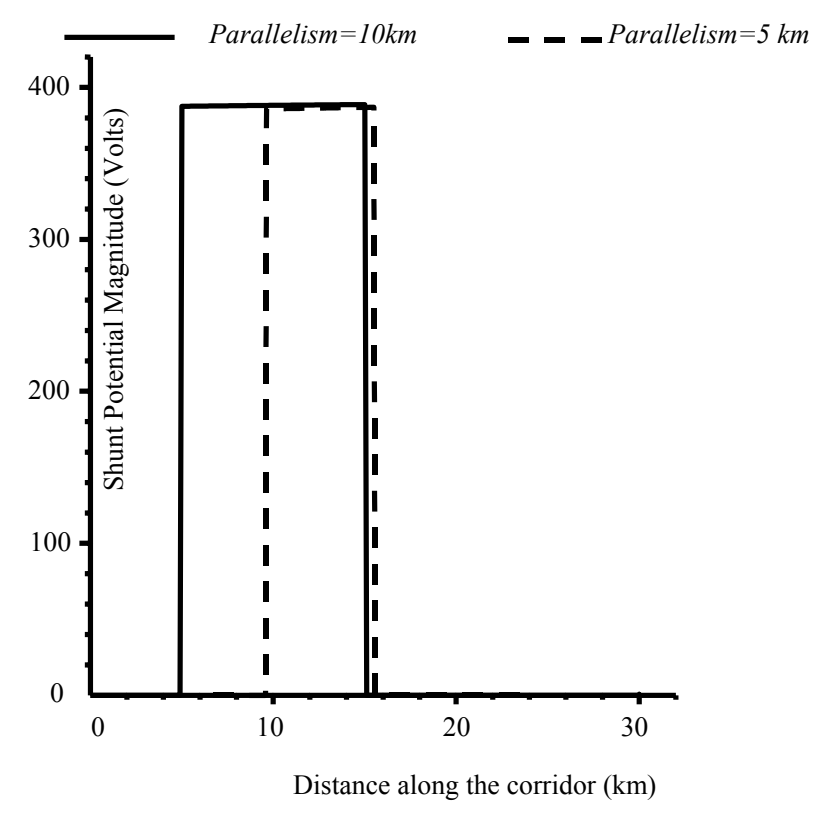

Fig. 15: Pipeline potential for $r=10 \mathrm{~m}, \mathrm{~V}=132 \mathrm{kV}, \rho=100$ $\Omega . m$ and for different parallelism.

\section{EARTHED PIPELINE ABOVE GROUND}

In this scenario a $10 \mathrm{~km}$ pipeline is considered which is run in parallel to a $30 \mathrm{~km}$ overhead transmission lines. The pipeline is maintained at a level of $0.5 \mathrm{~m}$ from the earth surface. Furthermore, the pipeline is earthed from both ends by grounding rods.

\section{A. Capacitive coupling}

Because the pipeline is earthed at both ends, the capacitive coupling is very small and can be neglected as shown in Fig. 16.

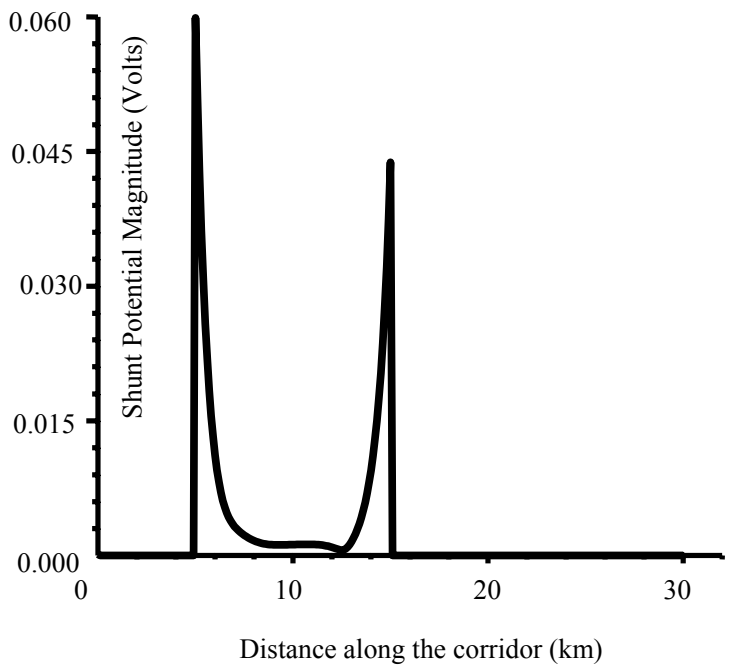

Fig. 16: Pipeline Potential for $\mathrm{r}=10 \mathrm{~m}, \rho=100 \Omega . \mathrm{m}$, Voltage $=132 \mathrm{kV}$ and pipeline earthed

\section{B. Inductive coupling}

As shown in Fig. 17, the induced voltage on the pipeline is reduced when the pipeline is connected to ground from both sides. However, this voltage is still more than the allowable limit in accordance with IEEE standard [8]. Therefore a mitigation system needs to be designed in order to bring this voltage down to a safe value $[3,7]$.

\section{Conductive coupling}

The maximum touch voltage is reduced to almost $50 \%$ when the pipeline is earthed from both sides compared with unearthed case as shown in Fig. 18. 


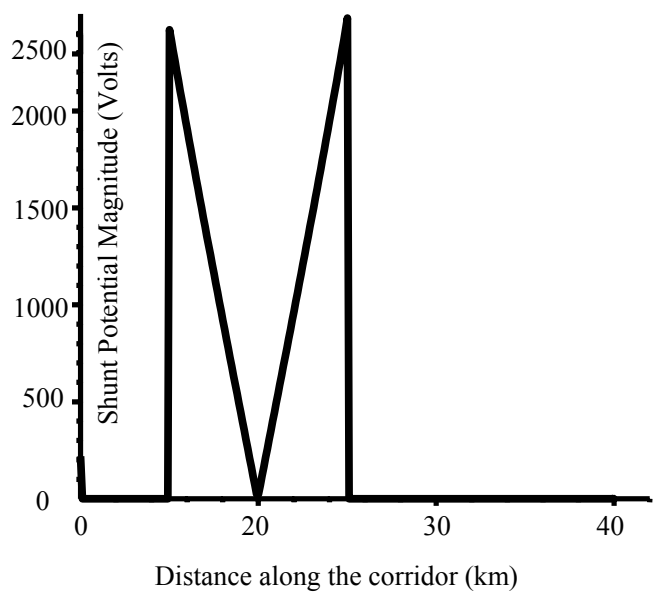

Fig. 17: Pipeline Potential, $r=100 \mathrm{~m}, \rho=100 \Omega . m$, $\mathrm{I}_{\mathrm{f}}=5 \mathrm{KA}$ and pipeline earthed

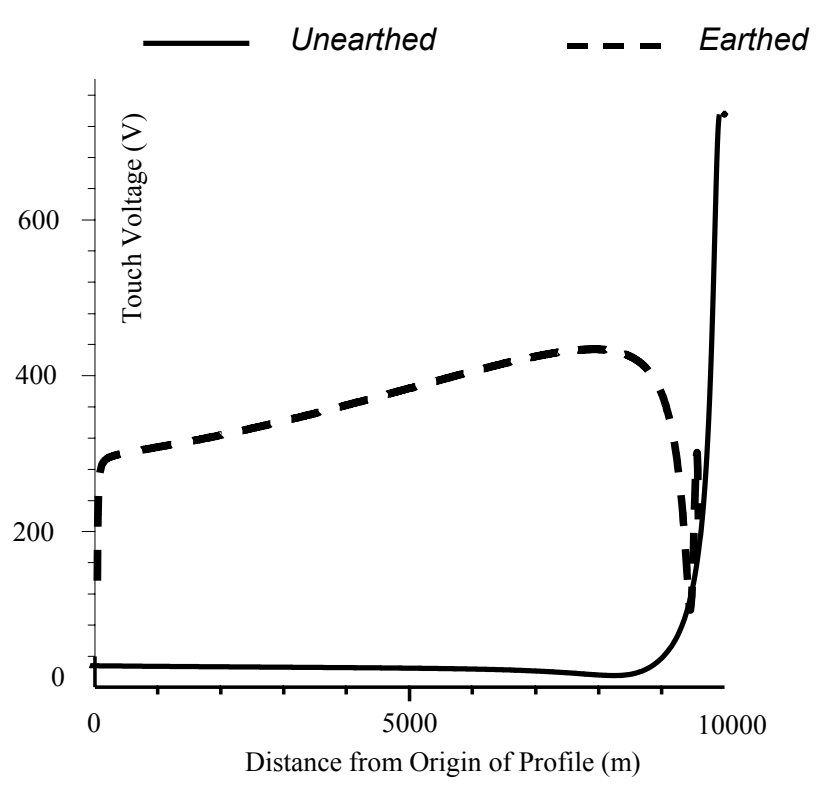

Fig. 18: Touch Voltage for $\mathrm{r}=100 \mathrm{~m}, \mathrm{I}_{\mathrm{f}}=5 \mathrm{kA}$ and $\rho=100 \Omega . m$

\section{CONCLUSIONS}

Inductive, conductive and capacitive coupling caused by a typical $132 \mathrm{kV}$ overhead transmission lines on neighboring 16inches diameter pipeline are investigated for three different scenarios of a pipeline. The model developed can predict the level of the voltage on the pipeline owing to each type of coupling. Results obtained from the software are further verified using well-known analytical equations. The results have shown that the voltage on the pipelines under fault conditions in such a power line are very large and are exceeding the acceptable limits determined in accordance to ANSI/IEEE Standard 80. The model developed has demonstrated the effects of various factors such as voltage, separation distance, current magnitude, soil resistivity and pipeline coating resistance on the interference levels. Some factors such as the fault current level, separation distance and soil resistivities were found to exhibit large influence on the pipeline voltage.

\section{REFERENCES}

[1] Y. Li, F. P. Dawalibi, and J. Ma: Electromagnetic Interference Caused by a Power System Network and a Neighboring Pipeline, Proceedings of the 62nd Annual Meeting of the American Power Conference, Chicago, April 10-12, 2000, pp. 311-316.

[2] CIGRE Working Group 36.02, “ Guide On The Interference of High Voltage AC Power Systems On Metallic Pipelines", 1995.

[3] R. D. Southey, F. P. Dawalibi, and W. Vukonich: Recent Advances in the Mitigation of AC Voltages Occurring in Pipelines Located Close to Electric Transmission Lines, IEEE Transactions on Power Delivery, Vol. 9, No. 2, April 1994, pp. 1090-1097.

[4] F. P. Dawalibi, R. D. Southey, J. Ma, and Y. Li: On the Mechanisms of Electromagnetic Interference between Electrical Power Systems and Neighboring Pipelines, NACE 2000, T10B Symposium on DC \&AC Interference, Orlando, March 26-31, 2000.

[5] Y. Baba, M. Ishii: Numerical electromagnetic field analysis on lighting surge response of tower with shield wire, IEEE Transactions on Power Delivery, Vol. 15, No. 3, July 2000, pp. 1010-1015.

[6] R. D. Southey, W. Ruan, and F. P. Dawalibi: AC Mitigation Requirements: A Parametric Analysis, The Corrosion/2001 NACE International Conference, Texas, March 11-16, 2001.

[7] Mitigation of Alternating Current and Lightning Effects on Metallic Structures and Corrosion Control Systems, NACE Standard RP0177-95.

[8] IEEE guide for safety in alternating current substation grounding (ANSI), Publication 80,1986.

[9] J. Dabkowski, M. Frazier: Power line fault current coupling to nearby natural gas pipelines, volume 3: analysis of pipeline coating impedance, EPRI Report EL-5472, A.G.A. Cat. No. L51537, August 1988.

[10] CDEGS Software Package Safe Engineering Services \& Technologies ltd., Montreal, Quebec, Canada, 2000.

[11] Y. Li, F. P. Dawalibi, J. Ma and R. D. Southey: Integrated Analysis Software for Electromagnetic Interference between Power lines and Neighboring Utilities, Proceeding of the International Conference on Electrical Engineering (ICEE, 2001), Xian, China, July 22-26, 2001.

[12] Y. Li, F. P. Dawalibi and J.Ma: Effect of Conductor Angle between Transmission Lines and Neighboring Utilities on the Accuracy of Inductive Interference Computations, International Conference on Power System Technology, PowerCon 2002, Vol. 1, China, October 13-17, 2002, pp. 98-105.

[13] F. P. Dawalibi and A. Selby: Electromagnetic fields of Energized Conductors, IEEE Transactions on Power Delivery, Vol. 8, No. 3, July 1993, pp. 1275-1284.

[14] A. Selby and F. P. Dawalibi: Determination of Current Distribution in Energized Conductors for the Computation of Electromagnetic Fields, IEEE Transactions on Power Delivery, Vol. 9, No. 2, April 1994, pp. 1069-1078.

[15] "Electrical Transmission and Distribution Reference Book" $4^{\text {th }}$ Ed., Westinghouse, Electric Corp., East Pittsburgh, PA, 1964. 


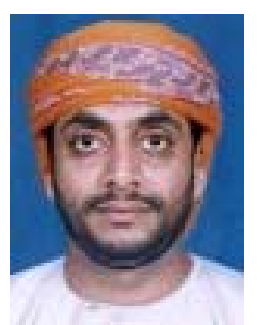

Abdullah H. Al-Badi obtained the degree of B.Sc. in Electrical Engineering from Sultan Qaboos University, Oman, in 1991. He received the degree of M.Sc. and Ph.D from UMIST, UK, in 1993 and 1998 respectively. September 1991, he joined the Sultan Qaboos University as demonstrator and, in 1998, he became an Assistant professor. His areas of interest include modeling and simulation of electrical machines, drives and interferences. He has published several papers in International Journals and Conferences in the filed of electrical machines, drives, interference and high voltage. He carried out several projects on electrical machines and the effect of $\mathrm{AC}$ interferences on pipelines. $\mathrm{He}$ is a Member of the Institute of Electrical Engineering and Electronics, IEEE, USA.

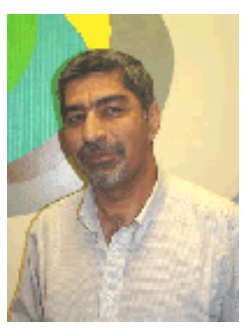

Hussain M. Al-Rizzo obtained his Ph.D. in Electrical Engineering (Highest Honors) on January 1992 from the Department of Electrical and Computer Engineering, University of New Brunswick, Fredericton, NB, Canada. He is currently an Associate Professor of Systems Engineering at the Donaghey College of Information Science and Systems Engineering, University of Arkansas at Little Rock, Little Rock, AR, USA. His research areas are in the fields of ComputerAided Design (CAD) of Guided Waves Components and Antennas, Electromagnetic Wave Propagation, Electromagnetic Interaction and Scattering in Complex Media, GPS, Industrial High-Power Microwave Heating Applications, Smart Antennas for Wireless Communications, MIMO Wireless Communications, Design of Antennas and Wireless communications Systems for Implantable Medical Devices, and Dynamic Channel Allocation and Load Balancing for Access Points in WLAN.

\section{Appendix A}

\section{Coupling Factors for Magnetic Induction}

Low frequency coupling depends upon the physical configuration of the circuits and their separation, and for earth return circuit, also on the soil resistivity.

Fig. A shows a single-phase circuit consisting of a single conductor a, grounded at its far end with an earth return path. To illustrate the mutual effects produced by current flowing in the single-phase circuit, a second conductor $b$ is presented. As a result of Carson's formulas and using average heights of conductors above ground, the mutual impedance $Z_{a b}$ between conductors $\mathrm{a}$ and $\mathrm{b}$ with common earth return path may be written as [5]:

$$
Z_{a b}=\left(\frac{f}{60}\right) \cdot 0.621 \cdot\left[0.0954+j 0.2794 \cdot \log \frac{D_{e x}}{D_{a b}}\right]
$$

where:

$Z_{a b}$ : The mutual impedance between conductors $\mathrm{a}$ and $\mathrm{b}$ $\Omega / \mathrm{km}$
$D_{e x}$ : The depth of earth return path, $\mathrm{m}$

$D_{a b}$ : The separation between conductors a and $\mathrm{b}, \mathrm{m}$.

The depth of earth return path is given as

$$
D_{e x}=660 \cdot \sqrt{\frac{\rho}{f}}
$$

where:

$\rho$ : The earth resistivity, $\Omega . m$.

$f$ : The system frequency, $\mathrm{Hz}$

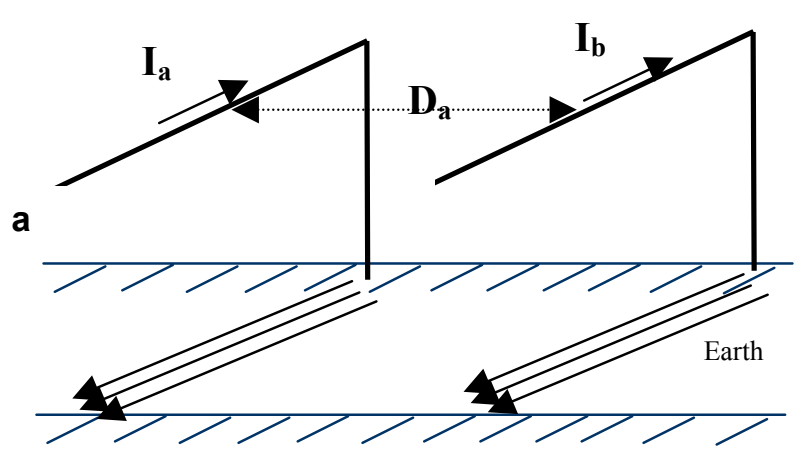

Fig. A: Single conductor single phase circuit with earth return

The voltages induced in a pipeline $\mathrm{x}$ caused by current $I_{a}$ flowing in a single earth-return circuit, illustrated in Fig. B, can be determined from the following approximate formula:

$$
V_{x}=I_{a} \cdot\left(\frac{f}{60}\right) 0.621 \cdot\left[0.0954+j 0.2794 \cdot \log \frac{D_{e x}}{D_{a x}}\right]
$$

where:

$V_{x}$ : The voltage induced in pipeline $\mathrm{x}, \mathrm{V} / \mathrm{km}$

$D_{a x}$ : The separation between conductor a and pipeline, $\mathrm{m}$.

$I_{a}$ : The r.m.s. value of current flowing in conductor a and return in earth, $\mathrm{A}$.

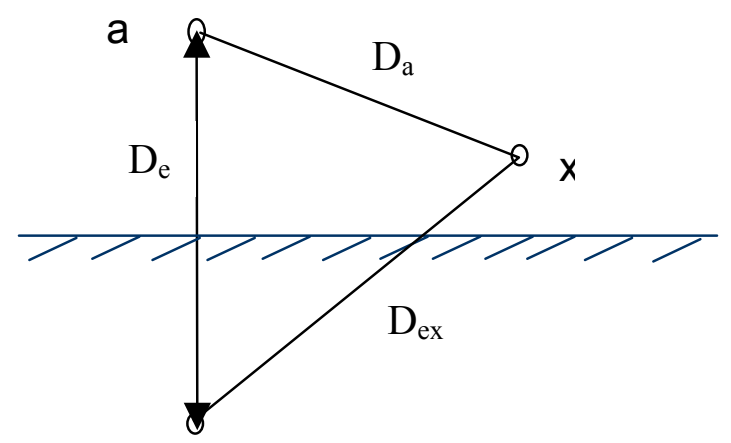

Fig. B: Induction from earth-return current 